\title{
Regulation of Protein-Carbohydrate Interactions in Bacteria and Fungi
}

\author{
Chukwuma C* \\ Centre for Future-Oriented Studies, Abakaliki, Ebonyi \\ State, Nigeria \\ *Correspondling author: Chrysanthus Chukwuma Sr, \\ Centre for Future-Oriented Studies, Abakaliki, Ebonyi \\ State, Nigeria
}

Received: December 02, 2016; Accepted: J anuary 23, 2017; Published: January 25, 2017

\begin{abstract}
This paper concerns protein-carbohydrate interactions as they undergird numerous significant biological activities with special emphasis on bacteria and fungi. Carbohydrates are relevant biopolymers which possess diverse functions. Predominantly, carbohydrates serve as recognition macromolecules by specifically recognizing other biomolecules. Lectins are proteins which bind carbohydrate structures. Protein-carbohydrate interactions constitute the foundation of specific carbohydrate recognition by lectins. In comparison to the research on protein-protein and protein-nucleic acid interactions, it is recently that scientists became interested more in protein-carbohydrate binding. Several of these interactions are associated with carbohydrates located at the cell surface as component of a membrane glycoprotein or glycolipid. Carbohydrate chains of glycoproteins, proteoglycans, polysaccharides, and glycolipids mediate multiple biological processes via their interactions with carbohydrate specific recognizing and binding proteins. These interactions are capable of influencing cellular adhesion and other cellular recognition activities, and also include signal transduction, inflammation, and host-pathogen recognition.
\end{abstract}

Keywords: Regulation; Bacteria; Fungi; Biochemical; Biosynthetic; Pathways; Proteins; Carbohydrates

\section{Introduction}

Numerous investigation activities have focused on nucleic acids, proteins and their mutual interactions as the paramount entities which govern the storage and transmission of information in living organisms [1]. However, carbohydrates have emerged as pre-eminent actors in the translation of encoded information into functional activity as demonstrated by their expansive encoding capacity and capability as well as the increasing significance in proteinmediated recognition events which result in biological elicitations. Carbohydrate information dissemination or messages encoded in free oligosaccharides, glycoproteins and glycolipids undergo interpretation by numerous lectins and other glycan-binding receptors which cause their translation into cellular activity. The inextricable linkage of protein-carbohydrate interactions in stringent and vital human health and disease, such as regulation of growth, tumour cell adhesion, cell migrations or host-pathogen recognition has augmented research interests in diverse areas. Structural methodologies have permeated the geometry of protein-carbohydrate interactions at the atomic instance which have provided the latitude for the clinical targeting of protein-carbohydrate interactions [2].

Protein-carbohydrate interactions constitute the crux of several vital biological processes as well as signaling, recognition and catalysis. A perspective of these interactions at the molecular level $[1,2]$ is liable to assist to develop newfangled, effective, efficient, increasingly predictive and selective therapeutic strategies which are potentially beneficial to mankind. Glycosidases and glycosyltransferases are certain carbohydrate-processing enzymes which govern the synthesis and breakdown of oligosaccharides. These enzymes have emerged as crucial targets in curbing bacterial and fungal pathogenesis, AIDS, and carcinogenic perturbations. The phenomena of binding and recognition are important processes whereby the body influences complex biological functions [3]. For instance, heparin presents ardent interest as an essential pharmaceutical product revealing the mechanisms of cross-reactivity which are aetiological agents in life-threatening side effects with the provision to develop a plethora of selective anti-clotting agents. Major targets for therapeutic intervention, such as the interactions of bacterial toxins with cell-surface receptors are certain binding processes mediated via multivalent protein-carbohydrate interactions [4]. A plausible research area involves carbohydrates since they are associated with essential biological processes. Carbohydrates have been demonstrated to be information carriers with identical or much increased relevance than amino acids [1] and nucleic acids [2].

\section{Fungi-Bacteria Interactions}

Ectomycorrhizal fungi are encompassed by bacterial communities with which they have metabolic and physical interactions during life cycle progression. These bacteria have the potential to present beneficial or deranging impacts on the production and activity of ectomycorrhizal. There is a paucity of data on the interaction mechanisms between ectomycorrhizal and associated bacteria. Comparative analyses of the pairwise transcriptome responses of the ectomycorrhizal fungus, Laccaria bicolor (Basidiomycota: Agaricales) in conjunction with beneficial, neutral and degradative soil bacteria revealed that the fungus responded differently to a specific bacterial strain distinctively [5]. Also, every bacterial strain formed a specific and unique response elicited in the fungal presence. Irrespective of these disparities in elicited responses at the gene level, it was possible to detect common gene classes associated with cell-wall interactions, 
stress response and metabolic progression linked to the interaction of the four microorganisms as shown by the comparative analyses of the three transcriptomes [5].

\section{Biophysical Methods in Structure Analyses}

The available information on molecular recognition of sugars by lectins including other carbohydrate-binding proteins can been used to develop new biomedical approach for strategic modeling. Biophysical techniques including X-ray crystallography and NMR spectroscopy are currently paramount in investigation activities on this discipline [2]. X-ray morphologies of protein carbohydrate complexes have revealed well-established functions of proteincarbohydrate interactions, such as stabilization via a network of hydrogen bonds usually associated with water molecules. The significance of these interactions has been examined in a few instances employing site-directed mutagenesis investigations [6]. A vast majority of well-refined $\mathrm{x}$-ray protein structures complexed with carbohydrates have increased. These structures have contributed significantly in elucidating the attributes of the atomic interactions between proteins and carbohydrates [6].

Also, Carbohydrate-Binding Molecules (CBMs) depict varied specificity and structure [7], thus positing a refined classification of CBM types. It is indicated that metals are involved in several roles in CBM function; and CBMs are liable to influence substrate specificity. A study of over twenty-six fungal lectin structures demonstrated inter alia binding to non-self glycans with resultant toxicity on predators, interactions of pathogenic fungi and host glycans, as well as the availability of natural and engineered lectins in biotechnology and research [8].

\section{Enzyme Mechanisms in Protein- Carbohydrate Interactions}

It has been demonstrated that glycoside hydrolases and polysaccharide lyases cleave glycosidic bonds, thus resulting in the formation of several novel enzymatic structures exerting control of diverse carbohydrates with a plethora of the families presenting unique structures. Certain of these enzymes posit representative catalytic residues, such as the Asp-His dyad. Newfangled methods have been employed to research the dynamics or activities of these enzymes [9-11]. There has been an augmentation in the series of solved structures of glycoside hydrolases and glycosyltransferases with their constitutive modules. These structures reveal that inasmuch as glycoside hydrolases depict an extraordinary variety of folds, glycosyltransferases and carbohydrate binding molecules ostensibly belong to a much decreased number of folding families [12]. In a research related to wall fortification per the synthesis, regulation and degradation of bacterial peptidoglycan, it was revealed that PBPs made use of the PG precursor molecule lipid II to augment new substance to the growing sacculus. PG synthesis was inextricably controlled at multiple stages. Bacteria have evolved vital PG modifications in order to evade the host immune system. Bacteria present diverse PG hydrolases which are necessary for bacterial cell morphogenesis. Profound PG recycling pathways make provision for re-ingress of released ingredients into PG biosynthetic pathway [13].

$\mathrm{N}$-acetylglucosamine-1-phosphate uridyltransferase (Gimu) exhibits the properties of a bifunctional enzyme that catalyses both the acetylation of glucosamine 1-phosphate and uridylation of $\mathrm{N}$-acetylglucosamine 1-phosphate, as well as two concomitant levels in the UDP-N-acetylglucosamine synthesis pathway in bacteria [14]. It is proposed that the 456 -amino acid $(50.1 \mathrm{kDa})$ protein may contain disparate uridyltransferase (N-terminal) and acetyltransferase (C-terminal) domains; which is confirmed via expression of the two distinct folding and functional domains. A fragment having the N-terminal 331 amino acids $(\operatorname{Tr} 331,37,1 \mathrm{kDa})$ possess only uridyltransferase activity, with steady state kinetic parameters identical to the full-length protein. Each of the bifunctional activities of the enzyme is important for cell viability. Investigation of truncated Gimu proteins by gel filtration localized regions of protein involvement in its trimeric stance. It was demonstrated that the acetyltransferase activity of the full-length protein is suppressed when embedded within heterotrimers produced in the presence of the truncated protein; thus, suggesting that the enzymatic activity necessitates a trimeric organization, with the catalytic site involved in contact sites between adjacent monomers. It was demonstrated that trimerization was perspicuously necessary for glucosamine-1phosphateacetyltransferase activity and cell growth [14].

The Saccharomyces cerevisiae protein Cks1 (cyclin-dependent kinase subunit 1) is important for the progression of cell-cycle. The biological activity of Cks1 is switch-modulated between two disparate molecular assemblies, viz, a single domain fold that emanates from the occlusion of a beta-hinge motif, and the intersubunit beta-strand interchanged dimer that generates from the orifice of the betahinge motif [15]. The Csk1 structure depicts conservation of betainterchanged dimer and the anion-binding site in yeast and human Cks during evolution. Thus, the Cks1 structure is suggestive of a biological role for the beta interchanged dimer and the anion-binding site to target Cdks to specific phosphoproteins during the progress of cell cycle.

It was shown that the yeast enzymes associated with UDP-GlcNAc biosynthesis are predictive targets for antifungal agents, such as GNA1 that is a new member of the Gcn5-related N-acetyltransferase (GNAT) superfamily, is involved in UDP-GlcNAc biosynthesis by the catalysis of the generation and production of GlcNAc6P from AcCoA and GlcN6P. Three crystal morphologies of the Apo Saccharomyces cerevisiae GNA1, GNA1-AcCOA, and GNA1-CoA-GlcNAc6P complexes were respectively refined to $2.4,1.3$, and 1.8 Angstrom resolution [16]. Investigation shows that these structures were not only stable, beta-intertwined, dimeric assembly with the GlcNAc6P binding region contained at the dimer interface, but also revealed the catalytic machinery of GNA1 at an atomic presentation. These results broaden comprehension of structural parameters necessary for GNAT activity, and depict morphological details for corresponding aminoglycoside $\mathrm{N}$-acetyltransferases, and the adaptability of the GNAT superfamily members to inculcate diverse specificities.

Trehalase ( $\mathrm{T}$ ) is an enzyme that is linked to energy metabolism and possesses regulatory action to harness trehalose concentrations in cells. Trehalase is associated in decreasing trehalose levels on stress alleviation. There are stipulations that the enzymes associated with trehalose synthesis (TPS, TPP) are complexed together and increasingly regulated at the activity and genetic levels of yeast. Trehalose that is present in bacteria, fungi and other organisms is a non-reducing disaccharide wherein both glucose components are in 
an a,a-1,1-glycosidic inextricable linkage as a source of energy and carbon. It may function as a signaling molecule to influence or regulate certain metabolic pathways or even to impact on growth, as well as protection of proteins and cellular membranes from inactivation or denaturation due to diverse stress situations [17]. Trehalose also constitutes an integral ingredient of several glycolipids which are significant cell wall structures. There are extant three distinct pathways for the biosynthesis of trehalose: (a) glucose transfer from UDPglucose to GDP-glucose to glucose-6-phosphate and UDP catalysed by the trhalose $-\mathrm{P}$ synthase with a trehalose-P-phosphatase that converts the trehalose-P to free trehalose. Another pathway involves certain unique bacteria linked to the intra-molecular rearrangement of maltose, glucosyl-a1,4-glucopyranoside in the conversion of the 1,4-linkage to the 1,1-trehalose bond catalysed by trehalose synthase, and culminates in free trehalose as the initial molecular product. A third pathway is associated with diverse enzymes wherein the first rearranges the glucose at the reducing end of a glycogen chain in the conversion of a1,4-linkage to an a,a1,1-bond; thereafter, another enzyme releases trehalose disaccharide from reducing end of the glycogen biomolecule. Furthermore, in mushrooms, trehalose phosphorylase catalyzes trehalose phosphorolysis in the formation of glucose-1-phosphate and glucose [17]. The reaction is reversible in vitro and is capable of producing trehalose from glucose-1-P and glucose.

There have been emergence of novel insights with respect to biologically and catalytic significant carbohydrate metal interactions [18]. It has been demonstrated that polysaccharide mono-oxygenases are copper-containing enzymes. It was observed that disparate sequence families in bacteria and fungi are structurally identical, but present surreptitious variations in mechanism and active site. The copper active sites employ $\mathrm{N}$-terminal histidine to coordinate to copper in the "histidine brace" nomenclature. Undergirding these enzymes and activities, refractory biomass presents as a potential biofuel source [19]. Carbohydrate flexibility and ring opening have been seen as vital processes in catalysis as determined by the morphologies of neuraminidase and xylose isomerase [20] and via the regulation by mRNAs [21].

\section{Host-Pathogen Elicitations}

Naturally, plants are invariably subjected to a plethora of disparate pathogens, such as bacteria, fungi and viruses. The interaction with certain of these organisms can be beneficial or harmful to the plant, as the event predicts [22]. In elucidating the mechanisms which are important in host-pathogen relationships, it is pertinent to consider (a) carbohydrate-binding proteins associated with host-pathogen interactions; (b) glycosyltransferases catalyzing mycobacterial cell wall synthesis; (c) protein-engineered lectins; and (d) biomolecular interactions. In comparing the lectin-binding attributes for predominantly dense beta-cyclodextrin-centred homo- and heteroglycoclusters with precise architecture makes provision as to extant evidence on inextricably linked synergic impacts (heterocluster effect) on carbohydrate-protein recognition scenario [23]. Plants are invariably subjected to environmental microorganisms, thus resulting in the evolution of complex mechanisms for their recognition and defence against potential pathogenic organisms. An instance of these elicitations is the downregulation of photosynthesis and several processes connected with primary metabolism necessary for plant growth. It is suggested that the energy conserved by downregulation of primary metabolism is diverted and employed for defense elicitations. Numerous studies have demonstrated that upregulation of primary metabolism is also evident during plant-pathogen interactions. It has been posited that upregulation of primary metabolism modulates signal transduction cascades which result in plant defence reactionary mechanisms. Due to pathogen or elicitor exposure, plants induce numerous genes connected with primary metabolic pathways, such as those associated with carbohydrate, amino acid and lipid syntheses and degradation. Genetic studies have also demonstrated associations of these metabolic pathways in plant defense elicitations [24]. The intricacies of plant defence responses necessitate appreciable amounts of energy which are essentially gathered from primary metabolic processes [25]. Primary metabolism is necessary for plant growth and development, thus genes associated with such processes are not liable to be easily eradicated by plant natural selection as is evident for R genes. Inasmuch as there is a plethora of evidence in the role of primary metabolism in plant defence responses, Studies are required for expansive elucidation and characterization of the underlying mechanisms and other components associated with such elicitations. Diseases which are resultant impacts of infections are generally due to specific interactions between a pathogen and the cells. Research on host-pathogen interactions has made provision for the drug design with therapeutic attributes.

Although, it has been observed in a few instances, microbe-host protein-carbohydrate interactions are fundamentally the initial step to precipitate infection. Enhanced glycotechnology is likely to aid in the identification of microbial carbohydrate binding proteins by employing affinity proteomics strategies. In certain instances, such conserved proteins may translate to viable vaccine ingredients; whereas in influenza, saccharide analogues could be the presenting choice [26]. Protein-carbohydrate interactions constitute massive potentials to develop and explore diverse biological processes which present crucial roles in cellular recognition, attachment and adhesion, particularly in host-pathogen interactions which culminate in infectious diseases since the surfaces of cells and pathogens exhibit complex carbohydrate structures and carbohydrate-binding proteins on their surfaces. Research has encompassed atomic basis of proteincarbohydrate interactions, morphology of Pseudomonas aeruginosa lectins, entobacterial infections of protein-carbohydrate interactions, retrocyclins as miniature lectins presenting potent antiviral action, C-type lectin receptors which regulate pathogen recognition via carbohydrate recognition mechanism, formulated carbohydratebased antimalarial vaccines, and glycobiology [27].

\section{Aspects of Protein-Carbohydrates Interactions in Future Research}

This study has embraced a perspective presentation of proteincarbohydrate interactions as they undergird numerous significant biological activities in the biosynthetic and biochemical pathways of bacteria and fungi. Carbohydrate chains of glycoproteins, proteoglycans, polysaccharidases, and glycolipids mediate multiple biological processes via their interactions with carbohydrate specific recognizing and binding proteins. Protein-carbohydrate interactions constitute the foundation of specific carbohydrate recognition by 
lectins. Carbohydrates are relevant biopolymers which possess diverse functions. Predominantly, carbohydrates serve as recognition macromolecules by specifically recognizing other biomolecules. Lectins are proteins which bind carbohydrate structures $[6,8]$. The localization of carbohydrates on the cell surface makes provision for their unique engagement with proteins on other cell surfaces or pathogens. Protein-carbohydrate interactions have been associated in varied physiological responses encompassing fertilization, development and immune system function. However, a specific protein-carbohydrate interaction is weaker than that of a proteinprotein interaction, as the former presents association constants ranging from 1000 - to $1,000,000$ fold more labile than the latter. To compensate and undergird their frailty and low affinity, a vast majority of protein-carbohydrate interactions are multivalent when multiple binding groups, as carbohydrates on a particular cell bind to multiple copies of a protein receptor of a corresponding cell [28-30].

In comparison to the research on protein-protein [1] and proteinnucleic acid [2] interactions, it is recently that scientists became interested more in protein-carbohydrate binding [31]. Several of these interactions are associated with carbohydrates located at the cell surface as component of a membrane glycoprotein, glycolipid or proteo-lipid [32]. These interactions are capable of influencing cellular adhesion and other cellular recognition activities. These also include inflammation, signal transduction, and host-pathogen recognition. In the effectuation of noncoding mRNA regulators, such as Spot42, Sgrs, GlmY, and G1m2 and a cis-encoded ribosome glms mRNA have been implicated in cellular pathways, stress responses and regulate sugar metabolism [21]. The study of these small RNA molecules contributed to the elucidation of the physiological implications, general principles and mechanisms of RNA-based regulation, such as post-transcriptional repression or activation of gene expression of polycistronic mRNAs, newfangled ribonucleoprotein complexes comprising smaller RNA Hfq and/or RNase E, as well as regulatory RNAs cascade [21]

Numerous biological recognition processes are associated with the binding and clustering of ligand-receptor complexes and resultant signal transduction. These interactions were observed in human $\mathrm{T}$ cells where binding and cross linking of specific glycoprotein counter-receptors on the cell surfaces due to an endogenous bivalent carbohydrate binding protein, galactin-1 results in apoptosis [33]. Diverse counter-receptors related to specific phosphatase or kinase activities were demonstrated to have produced disparate clusters on the cell surfaces due to galactin-1 binding to the carbohydrate moieties of the involved glycoproteins. It is suggested that the distinctive separation and organization of signaling molecules which emerge from galactin-1 binding is associated in concomitant signal dissipation. The galactin-1 potential in the elicitation to distinct glycoprotein receptors was parametrically modeled on molecular and structural research on multivalent carbohydrate binding to lectins with concomitant production of specific two- and threedimensional cross-linked lattices. These latter studies were depicted by $\mathrm{x}$-ray crystallographic results that one specific tetravalent lectin emerges as unique cross-linked complexes with four varied bivalent oligosaccharides [34]. It has been recently indicated in biological systems that binding and cross-linking of multivalent carbohydrates with multivalent lectins depict a new paradigm for supramolecular assembly and signal transduction [35]. Also, it has been recently suggested via isothermal titration calorimetry measurements of synthetic neoglycopolymers with Concanavalin A, Con A that heterofunctional diantennary architectures containing both alphamannose and non-binding beta-glucose units, poly(Man-Glc) binds to Con A; that the alpha-mannose bound to Con A facilitate interaction of beta-glucose with the extended binding site of Con A as a result of the presenting proximity of beta-glucose to alpha-mannose residues in the synthetic polymerizable scaffold [36].

It has been demonstrated that proteins which ingress the secretory pathway are translocated via the endoplasmic reticulum membrane in an unfolded state where they are limited to a quality control system that enables adroit folding or concomitant degeneration of inadequately folded polypeptides [37]. Mannose-trimming of N-glycans on recently synthesized proteins are significant in the recognition and sorting of terminally misfolded glycoproteins for ER-related protein degradation. Thus, misfolded proteins become retrotranslocated into the cytosol, polyubiquitinated, and consequently proteasomal degraded. Carbohydrate-dependent interactions cooperatively with $\mathrm{N}$-glycan-dependent interactions ostensibly regulate the intricate process of protein recognition and trajectory for proteosomal degradation. The quality control of recently produced secretory proteins is dependent on the potential of the endoplasmic reticulum to segregate and compartmentalize molecules in diverse folding forms. These folding presentations are directed by $\mathrm{N}$-glycans available on several secretory proteins. In ERAD, protein molecules which have been detected as terminally misfolded are consequently degraded at the cytosolic proteasome following dislodgment from the ER to the cytosol. Recent research suggests that these complex proteases are extremely dynamic, and are associated with transient assembly complexes of ERAD machinery [38].

\section{Discussion and Conclusion}

Bacteria and fungi reside cooperatively in expansive and diverse ambient, such as soils, plants, food products, animals and Man. The interactions between bacteria and fungi present remarkable impacts on their survival, colonization and pathogenesis. There are extant cases whereby bacteria make provision of compounds which augment the determinants of fungal virulence. Certain bacteria provide factors which are liable to be inhibitory to pathogenesis via the repression of fungal filamentation. Admixture of bacterial-fungal biofilms present attributes which are distinct from those exhibited by their singlespecies conformations. Clinical studies coupled with in vitro model systems are pertinent to unravel or elucidate the mechanisms which bacterial-fungal interactions effect human health and well-being $[39,40]$, and interspecies signaling as well as the implications of such signaling in the pathogenic expression of virulence traits between bacteria and fungi [41].

As carbohydrates are ubiquitous macromolecules which are involved in an exciting stance on the cell surface and within the cell [42]. This paper tends to explicate that through the augmented energetic contributions of multiple single complexes, multivalent interactions present kinetic lability, but display elevated ostensible affinity that complicates research on protein-carbohydrate interactions. Therefore, it becomes cumbersome to demonstrate the relevance and importance of protein-carbohydrate interaction in the 
assessment of the molecular mechanisms which are contributory to the production and undergirding of protein-carbohydrate complexes, as well as configure a paradigm or design for potent protein-carbohydrate interaction inhibitors, and synthesis $[37,43]$. In addition to the elucidation of these factors, it is pertinent for future studies to conduct more research on cell surface [42] glycogen composition and synthesis, cell-cell communities, hostpathogen interactions and therapeutics [44]; modification of cell wall biosynthetic pathways; application of biochemical, cellular and molecular as well as bioinformatics methods, biophysical techniques and microbial mutants; and the genes and enzymes related in the regulation $[37,43]$ of cell wall phenomena in response to environmental alterations. Nature utilizes posttranslational modifications to produce an expansive library of complex biological functions. The diverse repertoire of specific structures constitute a multitude of varied glycostructures demonstrable in organisms ranging from bacteria and fungi to man [2,4]. A variety of regulatory programmes in animal systems have been elucidated, revealing that carbohydrate-binding proteins are involved in cellular signaling via interaction with specific $\mathrm{N}$ - and $\mathrm{O}$ - glycans available on their moieties, components, or partners of interactions. The specific interaction and recognition between proteins and carbohydrates are usually depicted as initial steps in a sequence of activities. There is paucity of information regarding the significance of protein-carbohydrate recognition, but these interactions are perspicuous of relevance for growth and development as well as, defence reactions $[2,4,8]$, stress, and signaling.

\section{References}

1. Chukwuma C Sr. Control mechanisms of protein-protein interactions in biosynthetic pathways of bacteria and fungi. Applied Science Reports. 2016.

2. Fernandez-Alonso $M$ del C, Diaz D, Berbis MA, Marcelo F, Cañada J, Jiménez-Barbero J. Protein-carbohydrate interactions studied by NMR: from molecular recognition to drug design. Curr Protein Pept Sci. 2012; 13: 816830 .

3. Chukwuma C Sr. Perspectives on protein-nucleic acid interactions on the regulation of biosynthetic pathways in bacteria and fungi. J Adv Biol Basic Res. 2016

4. Williams SJ, Davies GJ. Protein-carbohydrate interactions: learning lessons from nature. Trends in Biotechnology. 2001; 19: 356-362.

5. Deveau A, Barret M, Diedhiou AG, Leveau J, de Boer W, Martin F. Pairwise transcriptomic analysis of the interactions between the ectomycorrhizal fungus Laccaria bicolor S238N and three beneficial, neutral and antagonistic soil bacteria. Microbial Ecology. 2015; 69: 146-159

6. Vyas NK. Atomic features of protein-carbohydrate interactions. Curr Opin Struct Biol. 1991; 1: 732-740.

7. Gilbert HJ, Knox JD, Boraston AB. Advances in understanding the molecular basis of plant cell wall polysaccharide recognition by carbohydrate-binding modules. Curr Opin Struct Biol. 2013; 2: 669-677.

8. Varrot A, Basher SM, Imberty A. Fungal lectins: structure, function and potential applications. Curr Opin Struct Biol. 2013; 23: 678-685.

9. Davies GJ, Henrissat B (Eds). Protein-carbohydrate interactions: Biophysical Methods. Curr Opin Struct Biol. 2013; 2: 649-794.

10. Davies GJ, Henrissat B. Cracking the code, slowly: the state of carbohydrate active enzymes in 2013. Curr Opin Struct Biol. 2013; 23: 649-651.

11. Fushinobu S, Alves VD, Coutinho PM. Multiple rewards from a treasure trove of novel glycoside hydrolase and polysaccharide lyase structures: new folds, mechanistic details, and evolutionary relationships. Curr Opin Struct Biol. 2013; 2: 652-659.
12. Bourne Y, Henrissat B. Glycoside hydrolases and glycosyltransferases: families and functional modules. Curr Opin Struct Biol. 2001; 11: 593-600.

13. Sobhanifar S, King DT, Strynadka NCJ. Fortifying the wall synthesis, regulation and degradation of bacterial peptidoglycan. Curr Opin Struct Biol. 2013; 2: 695-703

14. Pompeo F, Bourne Y, van Heijenoort J, Fassy F, Mengen-Lecreulx D. Dissection of the bifunctional Escherichia coli $\mathrm{N}$-acetylglucosamine-1phosphate uridyltransferase enzyme into autonomous functional domains and evidence that trimerization is absolutely required for glucosamine-1phosphateacetyltransferase activity and cell growth. J Biol Chem. 2001; 276: 3833-3839.

15. Bourne Y, Watson MH, Arval AS, Bernstein SL, Reed SI, Tainer JA. Crystal structure and mutational analysis of the Saccharomyces cerevisiae cell cycle regulatory protein Cks1: implications for domain swapping, anionbinding and protein interactions. STRUCT FD. 2000; 8: 841-850.

16. Peneff $C$, Mengin-Lecreulx D, Bourne $Y$. The crystal structures of apo and complexed Saccharomyces cerevisiae GNA1 shed light on the catalytic mechanism of an amino-sugar N-acetyltransferase. J Biol Chem. 2001; 276 16328-16334.

17. Elbein AD, Pan YT, Pastwzak I, Carroll D. New insights on trehalose: a multifunctional molecule. Glycobiology. 2003; 13: 17R-27R.

18. Chukwuma C Sr. Approaches to issues and concepts of heavy metals in biochemical and biosynthetic pathways. JBAH.2016.

19. Hemsworth GR, Davies GJ, Walton PH. Recent insights into coppercontaining lytic polysaccharide mono-oxygenases. Curr Opin Struct Biol. 2013; 23: 660-668.

20. Bourne $\mathrm{Y}$, van Tilbeurgh $\mathrm{H}$, Cambillau C. Protein-carbohydrate interactions. Curr Opin Struct Biol. 1993; 3: 681-686.

21. Gorke B, Vogel J. Noncoding RNA control of the making and breaking of sugars. Genes \& Dev. 2008; 22: 2914-2925.

22. Dangl JL, Horvath DM, Staskawicz BJ. Pivoting the plant immune system from dissection to deployment. Science. 2013; 341: 746-751.

23. Gomez-Garcia M, Benito JM, Rodriguez-Lucerna D et al. Probing secondary carbohydrate-protein interactions with highly dense cyclodextran-centered heteroglycoclusters: The heterocluster effect. J. Am. Chem. Soc. 2005; 127 : 7970-7971

24. Rojas CM, Senthil-Kumer M, Tzin V, Mysore KS. Regulation of primary plant metabolism during plant-pathogen interaction and its contribution to plant defense. Front Plant Sci, 2014; 5: 17.

25. Bolton MD. Primary metabolism and plant defense - fuel for the fire. Mol. Plant Microbe Interact. 2009; 22: 487-497.

26. Karlsson KA. Pathogen-host protein-carbohydrate interactions as the basis of important infections. Adv Exp Med Biol. 2001; 491: 431-443.

27. Bewley CA. Protein-carbohydrate Interactions in Infectious Diseases. RSC Biomolecular Sciences. 2006; DOI: 10:1039/9781847555335.

28. Kiessling LL, Pohl NL. Strength in numbers: non-natural polyvalent carbohydrate derivatives. Chem Biol. 1996; 3: 71-77.

29. Kiessling LL, Strong LE, Gestwicki JE. Principles for multivalent ligand design. Annu Rep Med Chem. 2000; 35: 321-330.

30. Strong LE, Kiessling LL. A general synthetic route to defined biologically active multivalent arrays. J Am Chem Soc. 1999; 121: 6193-6196.

31. Dwek RA. Glycobiology: Toward understanding the function of sugars. Chem Rev. 1996; 96: 683-720.

32. Chukwuma C Sr. Characterization of protein-lipid interactions in biochemical and biosynthetic pathways. JABBR.

33. Pace KE, Lee C, Stewart PL, Baum LG. Restricted receptor segregated into membrane microdomains occurs on human T cells during apoptosis induced by Galactin-1. J Immunol, 1999; 163: 3801-3811.

34. Olsen RL, Dessen A, Gupta D et al. X-ray crystallographic studies of unique 
cross-linked lattices between four isomeric biantennary oligosaccharides and soybeab agglutinin. Biochemistry. 1997; 36: 15073-15080.

35. Sacchettini JC, Baum LG, Brewer FC. Multivalent protein-carbohydrate interactions. A new paeadigm for supermolecular assembly and signal trasnsduction. Biochemistry. 2001; 40: 3009-3015.

36. Loka RS, McConnell MS, Nguyen ML. Studies of highly -ordered heterodian tennary mannose/glucose functionalized polymers and Concanavalin A protein interactions using isothermal titration calorimetry. Biomolecules. 2015; 16: 4013-4021.

37. Stominska-Wojewodzka M, Sandvig K. The role of lectin-carbohydrate interactions in the regulation of ER-associated protein-degradation. Molecules. 2015; 20: 9816-9846.

38. Benyair R, Ogen-Shtern N, Lederteremer GZ. Glycan regulation of ERassociated degradation through compartmentalization. Semin Cell Dec Biol. 2015; 41: 99-109.
39. Wargo MJ, Hogan DA. Fungal-bacterial interactions: a mixed bag of mingling microbes. Curr Opin Microbiol. 2006; 9: 359-364.

40. Peleg AY, Hogan DA, Mylonakis E. Medically important bacterialOfungal interactions. Nat Rev Microbiol. 2010; 8: 340-349.

41. Holcombe LJ, O'Gara F, Morrissey JP. Implications of interspecies signaling for virulence of bacterial and fungal pathogens. Future Microbiol. 2011; 6 : 799-817.

42. De Schutter K, van Damme EJM. Protein-carbohydrate interactions, and beyond. Molecules. 2015; 20: 15202-15205.

43. Wang Q, Groenendyk J, Michalak M. Glycoprotein quality control and endoplasmic stress. Molecules. 2015; 20: 13689-13704.

44. Chiodelli P, Urbinati C, Bugatti A, Rusnati M. Heparin/heparin sulfate proteoglycans glycomic interactome in angiogenesis: Biological implications and therapeutical use. Molecules. 2015; 20: 6342-6388.
J Bacteriol Mycol - Volume 4 Issue 1 - 2017

ISSN : 2471-0172 | www.austinpublishing group.com

Chukwuma. (C) All rights are reserved
Citation: Chukwuma C. Regulation of Protein-Carbohydrate Interactions in Bacteria and Fungi. J Bacteriol Mycol. 2017; 4(1): 1041 